Exp. Anim. 55(1), 49-56, 2006

\title{
Increased Susceptibility to Staphylococcus aureus Colonization of the Skin of the NOA mouse: A Potentially Useful Animal Model for Evaluating Antiseptic Effects
}

\author{
Taizo KONDO'), Hitoshi OHNO ${ }^{2)}$, Keisuke TAGUCHI'1), Ryotaro SATODE1), \\ Toshio KONDO'1), and Yasuhisa SHIOMOTO ${ }^{1)}$
}

${ }^{1)}$ Division of Pharmacology, Drug Safety and Metabolism, and ${ }^{2)}$ Pharmaceutical Technology Institute,
Otsuka Pharmaceutical Factory, Inc., 115 Tateiwa, Muya-cho, Naruto, Tokushima 772-8601, Japan

\begin{abstract}
Isolation of bacteria from wet skin lesions was attempted using Naruto Research Institute Otsuka Atrichia (NOA) mice, which develop such lesions spontaneously at a high rate. As a result, Staphylococcus aureus was demonstrated to have colonized the wet skin lesions at high density. In addition, the isolated S. aureus was found to be similar to the strain of $\mathrm{S}$. aureus thought to colonize the eczematous lesions seen in humans with atopic dermatitis. Furthermore, a survey of the $\mathrm{S}$. aureus colonization status of NOA mice with no wet skin lesions confirmed colonization at higher density than in HR-1 mice as control, indicating that the skin of the NOA mouse has the novel characteristic of increased susceptibility to $\mathrm{S}$. aureus colonization. Thus, by using changes in $\mathrm{S}$. aureus counts as an index, the NOA mouse can be expected to serve as a useful animal model for evaluating the effects of topical antiseptics. The antiseptic effects of an ointment and a lotion containing chlorhexidine gluconate were confirmed using this animal model.
\end{abstract}

Key words: animal model, chlorhexidine gluconate, evaluating antiseptic effects, NOA mouse, Staphylococcus aureus

\section{Introduction}

The Naruto Research Institute Otsuka Atrichia (NOA) mouse is a hair-deficient mutant [8] that has been established as an inbred strain in our facility. Its most significant characteristic is the spontaneous development of ulcerative and erosive wet skin lesions (Fig. 1) at high rate. Such lesions are seen from the 10th week of age, with prevalence rates of $30 \%$ by the 10 th week of age and $90 \%$ by the 20th week of age [8]. Development of the skin lesions has also been observed in facilities other than our facility (all facilities supplied with the NOA mouse). Since the cleanliness class of these facilities was different each other, such lesions are considered to develop regardless of the cleanliness of facility.

(Received 5 August 2005 / Accepted 14 November 2005)

Address corresponding: T. Kondo, Division of Pharmacology, Drug Safety and Metabolism, Otsuka Pharmaceutical Factory, Inc., 115

Tateiwa, Muya-cho, Naruto, Tokushima 772-8601, Japan 


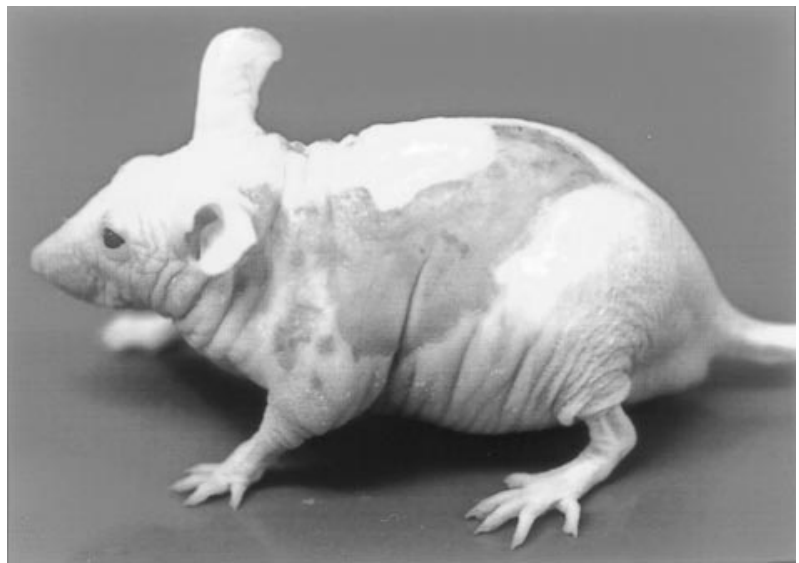

Fig. 1. Wet skin lesions on an NOA mouse (male, 25 weeks of age).

In our studies to date, we have identified the NOA mouse to be a mutant with various potential uses, particularly those listed below.

1) Since the involvement of mast cells and $\operatorname{IgE}[8]$ as well as eosinocytes [9] is suggested in the development and aggravation of wet skin lesions, the NOA mouse is expected to be a useful animal model of allergic dermatopathy in humans.

2) When NOA mice are maintained under different housing conditions, the time of onset and prevalence of wet skin lesions differ. Since psychosocial factors are thought to be involved in the development and aggravation of such skin lesions, the NOA mouse can be expected to serve as a useful animal model for analyzing the relationships between dermatopathy and psychological conditions [10].

3) When NOA mice were housed individually, dry skin developed in all animals [10], and a marked decrease was observed in the water content of the stratum corneum [11]. Applying ointment to the dry skin restored the water content in proportion to the oil content of the ointment. The moisturizing effect of urea was also confirmed. This animal model is therefore expected to be useful for evaluating the effects of topical moisturizers [11].

In a preliminary study, we found that Staphylococcus aureus colonized the wet skin lesions of NOA mice at high density. In the present study, to confirm this observation, bacteria were isolated from the wet skin lesions, the isolates were identified and subclassified, and the types of $S$. aureus colonizing the skin lesions were examined. Further, the $S$. aureus colonization status of the skin of NOA mice that did not develop skin lesions was surveyed, using HR-1 mice as control. Using the bacterial count as an index, we attempted to assess the usefulness of this animal model for evaluating the effects of topical antiseptics. The effect of topical antiseptics on the living body cannot be known from the results of in vitro studies alone, and no suitable animal model for in vitro assessment has been reported to date.

\section{Materials and Methods}

This study was carried out using SPF animals under a barrier system. Our facility has periodically been subjected to microbiological monitoring, and confirmed to be free of categories A, B, C, and E microbes and parasites specified for monitoring in mice and rats by the ICLAS Monitoring Center (Asia) (5). All of the NOA mice used were reared at our facility. The breeding conditions were described in our previous report [10].

Identified and subclassification of S. aureus colonizing wet skin lesions

Ten male animals (59-640 days of age) that developed wet skin lesions on the dorsal region (back, lumbar region, or lateral abdominal region) were selected from among the NOA mice maintained at our facility. To isolate the bacteria, samples were collected from the wet skin lesions using a sterilized swab moistened with sterile isotonic sodium chloride solution, smeared on Trypticase ${ }^{\circledast}$ Soy Agar II plates with 5\% sheep blood (Nippon Becton Dickinson Co., Ltd., Tokyo, Japan), and cultured at $37^{\circ} \mathrm{C}$ for $48 \mathrm{~h}$ under aerobic conditions. To obtain pure cultures, 5 colonies were selected for each animal from among the colonies growing in isolation cultures. They were smeared on salt egg yolk agar plates prepared using Salt Egg Yolk Agar Base "Nissui" (Nissui Pharmaceutical Co., Ltd., Tokyo, Japan), and cultured at $37^{\circ} \mathrm{C}$ for $48 \mathrm{~h}$ under aerobic conditions. Identification tests were performed for all bacterial isolates in the pure cultures using API STAPH (an identification system for staphylococci, micrococci, and related genera) in the API manual kit (bioMérieux SA, Marcy l'Etoile, France) for simple testing of bacteria. 
Isolates identified as $S$. aureus underwent subclassification tests for the following 4 items:

(i) Staphylococcus Coagulase Typing Test

(ii) Exfoliative toxin (ET) (A and B) producibility: EXT-RPLA

(iii) Staphylococcal enterotoxin (SE) (A, B, C, and D) producibility: SET-RPLA

(iv) Toxic shock syndrome toxin 1 (TSST-1) producibility: TST-RPLA

The reagents and kits used are produced by Denka Seiken Co., Ltd., Tokyo, Japan. Tests were performed according to the manufacturer's instructions.

Brain heart infusion broth prepared using Brain Heart Infusion (Becton Dickinson Company, Sparks, MD, USA) was used to prepare the samples (culture medium) for subclassification.

Survey of the S. aureus colonization status of the skin

Five, 5-week-old male NOA mice with skin of normal appearance were housed in 1 cage. TGSE agar, a 10-cm ${ }^{2}$ stamp-type $S$. aureus-selective agar (Food Stamp ${ }^{\circledR}$ "Nissui" TGSE Agar, Nissui Pharmaceutical Co., Ltd.), was used as the medium.

A TGSE agar stamp was pressed against the dorsal skin (back, lumbar region, and lateral abdominal region) of each animal, centering on the apex of the primary curvature of the vertebral column, to collect bacteria. Collection of bacteria was performed a total of 6 times at 2-week intervals up to 15 weeks of age. At the time of collection, the entire skin surface of each animal was visually examined. After collection, the medium was incubated at $37^{\circ} \mathrm{C}$ for $48 \mathrm{~h}$ under aerobic conditions. Black colonies surrounded by cloudy bands were counted as $S$. aureus colonies according to the manufacturer's instructions for the medium. A maximum of 5 colonies per animal were selected from among those showing these characteristics, and pure cultures were grown using salt egg yolk agar plates. These were then identified and subclassified.

Male HR-1 mice (Hos:HR-1) obtained from Hoshino Laboratory Animals (Saitama, Japan) at 4 weeks of age were used as controls and studied in a similar manner.

Assessment of the usefulness of the animal model for evaluating the effects of topical antiseptics

Fifty, 15-week-old male NOA mice with skin of normal appearance were used. They were housed at 5 animals per cage. The test substances were an ointment containing $0.2 \%$ chlorhexidine gluconate ( $\mathrm{CHG}$ ), its base, a lotion containing $0.05 \% \mathrm{CHG}$, and its base, for a total of 4 test substances. All test substances were prepared according to our in-house recipe. Each group consisted of 10 animals, with a no-treatment group serving as the control.

A TGSE agar stamp was pressed against the dorsal skin (back, lumbar region, and lateral abdominal region) of each animal, centering on the apex of the primary curvature of the vertebral column, to collect bacteria prior to the application of any of the test substances. The test substances were then applied to or sprayed onto the animals' dorsal skin. Approximately $0.02 \mathrm{~g}$ of ointment was used, an amount that could be spread thinly over the entire dorsal skin surface. The lotions were sprayed from a distance of about $15 \mathrm{~cm}$ onto the dorsal surface of animals suspended by the tail. About $0.2 \mathrm{~g}$ of solution was applied, an amount that would not show a washout effect. After application of the test substance, each animal was temporarily placed in individual housing. Bacteria were collected 3 $\mathrm{h}$ after application by pressing a TGSE agar stamp against the same site from where bacteria were collected before application. The media for collection of bacteria were incubated at $37^{\circ} \mathrm{C}$ for $48 \mathrm{~h}$ under aerobic conditions, black colonies surrounded by cloudy bands were counted, and the ratio of the number of colonies at $3 \mathrm{~h}$ after application to that before application was calculated.

The results obtained were subjected to the SteelDwass test for statistical analysis, and the bactericidal effect was evaluated. SAS software (SAS Institute Japan, Ltd., Tokyo, Japan) was used for statistical analysis.

The present study was approved by the Committee on the Care and Use of Laboratory Animals of Otsuka Pharmaceutical Factory, Inc.

\section{Results}

Identified and subclassification of S. aureus colonizing wet skin lesions

In isolation cultures, uniformly light-yellow colonies showing $\beta$-hemolytic activity were found at high densities in wet skin lesions of all 10 NOA mice. In pure cultures, all of the 50 isolates from the 10 animals 
Table 1. Results of subclassification of S. aureus isolated from wet skin lesions of NOA mice

\begin{tabular}{llc}
\hline Coagulase type & \multicolumn{1}{c}{ III } \\
\hline ETA & & Negative \\
\hline ETB & & Negative \\
\hline & SEA & Negative \\
\cline { 2 - 3 } & SEB & Negative \\
\cline { 2 - 3 } Superantigens & SEC & Positive \\
\cline { 2 - 3 } & SED & Negative \\
\cline { 2 - 3 } & TSST-1 & Negative \\
\hline
\end{tabular}

ETA: Exfoliative toxin A. ETB: Exfoliative toxin B. SEA: Staphylococcal enterotoxin A. SEB: Staphylococcal enterotoxin B. SEC: Staphylococcal enterotoxin C. SED: Staphylococcal enterotoxin D. TSST-1: Toxic shock syndrome toxin 1 . yielded yellow colonies showing positive egg yolk reactions. In identification testing, all 50 of the selected isolates were judged to be $S$. aureus.

In the subclassification test, the results obtained for the 50 isolates were all the same, as shown in Table 1. Specifically, every isolate was coagulase type III, producing staphylococcal enterotoxin C (SEC), but not ET or TSST-1.

Survey of the S. aureus colonization status of the skin

The results of the $S$. aureus colony counts in the NOA mice are shown in Table 2, and the results in HR1 mice are shown in Table 3 . The average number of colonies of $S$. aureus per agar plate was 5.2 for 5week-old NOA mice and 130.6 for 15 -week-old NOA mice, whereas the corresponding figures for HR-1 mice were 1.2 and 0.0 , respectively. Throughout the test period up to 15 weeks of age, the external appearance of the skin was normal in all of the animals.

The total number of isolates that underwent pure cul-

Table 2. Changes in the number of colonies of S. aureus on the dorsal skin of NOA mice

\begin{tabular}{ccccccc}
\hline Animal No. & 5 weeks & 7 weeks & 9 weeks & 11 weeks & 13 weeks & 15 weeks \\
\hline 1 & 3 & 12 & 33 & 102 & 141 & 168 \\
2 & 17 & 41 & 46 & 137 & 23 & 133 \\
3 & 3 & 9 & 29 & 38 & 33 & 118 \\
4 & 3 & 56 & 60 & 15 & 47 & 83 \\
5 & 0 & 182 & 184 & 109 & 100 & 156 \\
\hline MEAN & 5.2 & 60.0 & 70.4 & 80.2 & 68.8 & 130.6 \\
\hline SD & 6.7 & 41.0 & 64.7 & 51.4 & 50.1 & 32.1 \\
\hline
\end{tabular}

Number of colonies per agar plate.

Table 3. Changes in the number of colonies of S. aureus on the dorsal skin of HR-1 mice over time

\begin{tabular}{ccccccc}
\hline Animal No. & 5 weeks & 7 weeks & 9 weeks & 11 weeks & 13 weeks & 15 weeks \\
\hline 1 & 0 & 24 & 7 & 0 & 2 & 0 \\
2 & 0 & 7 & 0 & 0 & 1 & 0 \\
3 & 2 & 3 & 0 & 1 & 2 & 0 \\
4 & 4 & 5 & 1 & 0 & 0 & 0 \\
5 & 0 & 47 & 0 & 0 & 0 & 0 \\
\hline MEAN & 1.2 & 17.2 & 1.6 & 0.2 & 1.0 & 0.0 \\
\hline SD & 1.8 & 18.6 & 3.0 & 0.4 & 1.0 & 0.0 \\
\hline
\end{tabular}

Number of colonies per agar plate. 


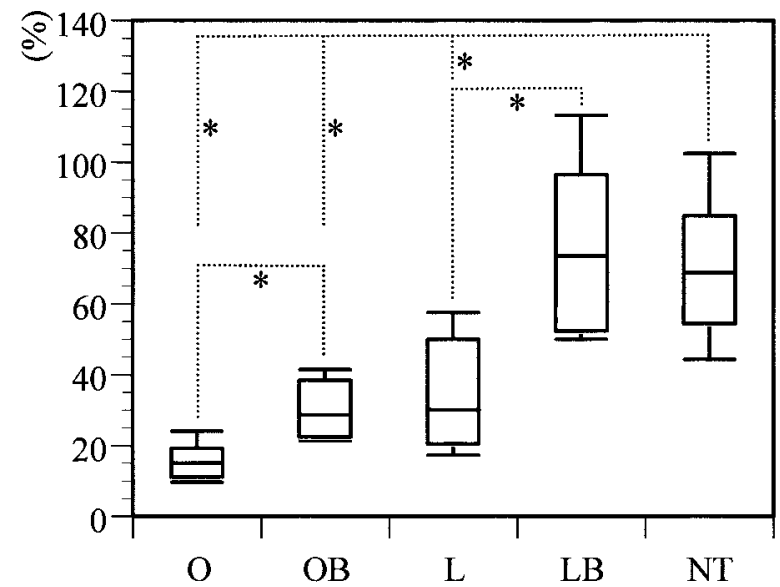

Fig. 2. Bactericidal effects of chlorhexidine gluconate. Ordinate: Ratio of the number of $S$. aureus colonies $3 \mathrm{~h}$ after application to the number before application (\%). O: Ointment containing $0.2 \%$ CHG. OB: Ointment base. L: Lotion containing $0.05 \%$ CHG. LB: Lotion base. NT: Non-treated. $\mathrm{n}=10$ for each group. *: Significant difference $(P<0.05)$.

turing, identification, and subclassification was 139 for NOA mice and 41 for HR-1. The identification and subclassification results for all isolates were the same as those obtained for $S$. aureus colonizing the wet skin lesions of NOA mice.

Assessment of the usefulness of the animal model for evaluating the effects of topical antiseptics

The effects on S. aureus colony counts of the application of the ointment and the spraying of the lotion are shown in Fig. 2. The ratio of the number of colonies before and after application was $15.7 \%$ for the ointment containing CHG (O), 29.8\% for the ointment base (OB), $35.0 \%$ for the lotion containing $\mathrm{CHG}(\mathrm{L})$, and $77.5 \%$ for the lotion base (LB). Significant differences $(P<0.05)$ were found between $\mathrm{O}$ and $\mathrm{OB}$ and between $\mathrm{L}$ and LB.

\section{Discussion}

The isolation and identification results for bacteria obtained from the wet skin lesions of NOA mice show that $S$. aureus colonized the skin lesions at high density. Furthermore, the results of subclassification show that the $S$. aureus colonizing the lesions was a coagulase type III, SEC-producing strain (Table 1). In the present study, animals that developed wet skin lesions in the dorsal region during rearing were selected. This was due to concerns regarding the possible effects on the test results of exposure of the skin lesions in the ventral region to feces or other contaminants. It cannot be judged from the results of the present study alone whether or not other bacterial species colonize the wet skin lesions of NOA mice.

It is known that $S$. aureus is detected at a high rate in the skin of patients with atopic dermatitis (AD), a typical allergic dermatopathy in humans, and that this species colonizes chronic eczematous lesions at a particularly high density [12]. In addition, S. aureus has long been recognized as an aggravating factor in $\mathrm{AD}$ $[1,2]$. The fact that patients with $\mathrm{AD}$ are known to have high ratios of antibodies against the superantigens produced by $S$. aureus in the blood also suggests the involvement of $S$. aureus in AD [15]. The S. aureus that colonizes the eczematous lesions in patients with $\mathrm{AD}$ has been reported to be a coagulase type III or VII strain that produces superantigens [13] but not ET [6]. In contrast, the $S$. aureus that is responsible for primary dermal infections such as staphylococcal scalded skin syndrome (SSSS) and impetigo contagiosa is known to be a coagulase type I or V strain that produces ET [6], and it is therefore considered to be possible to distinguish this strain from the $S$. aureus that colonizes eczematous lesions in AD. Accordingly, the S. aureus strain that colonizes the wet skin lesions of NOA mice at high density appears to be similar to the strain that colonizes the eczematous lesions in humans with AD. In NOA mice, although development of skin lesions was also observed in the germ-free animals, the lesions were slight compared with SPF animals (unpublished data). Therefore, it is considered that S.aureus is associated with aggravation of the lesions. Consequently, it is thought to be worthwhile to examine the relationships between wet skin lesions and $S$. aureus in the NOA mouse and to investigate the possible usefulness of the present animal model for analyzing the relationships between human $\mathrm{AD}$ and $S$. aureus.

According to the survey results of the S. aureus colonization status in the skin of NOA mice in which no wet skin lesions developed (Table 2), the density of $S$. aureus colonizing the skin of NOA mice increases with age. The colonization density differed among individual animals when they were young, even when they were 
housed in the same cage, so individual differences are thought to decrease with advancing age. In addition, when compared with hairless $(h / h)$ mutant HR-1 mice (Table 3), the greater tendency of the skin of NOA mice to become colonized with $S$. aureus is obvious. It is clear that the water content of the stratum corneum and the sebum quantity on the skin surface are lower and that the $\mathrm{pH}$ value on the skin surface is higher in NOA mice than in HR-1 mice [11], and these dermal characteristics are thought to contribute to the susceptibility of the skin of NOA mice to colonization with $S$. aureus.

From the results of the subclassification testing of $S$. aureus isolated from the skin of NOA mice in which no wet skin lesions developed, it was shown that $S$. aureus that colonizes normal skin of NOA mice possesses the same characteristic as the isolates from wet skin lesions in NOA mice. S. aureus that colonizes HR-1 mouse skin showed the same characteristics as well.

The present study was conducted using SPF animals under a barrier system. S. aureus is not included in the specific pathogens designated for our facility. Basic studies on the occurrence of $S$. aureus in the rearing environment in our facility and the animals themselves provided the following findings (unpublished data).

1) S. aureus other than the type that colonizes the skin of the NOA mouse is also present in the breeding environment.

2) The $S$. aureus isolated from the fur of normal mice is the same type as that which colonizes the skin of the NOA mouse.

3) The $S$. aureus isolated from the skin of hairless $(h /$ $h$ ) and nude $(n u / n u)$ mice is the same type as that which is isolated from the NOA mouse.

4) The S. aureus isolated from preputial gland abscesses in mice is the same type as that which colonizes the skin of the NOA mouse.

5) The $S$. aureus isolated from dermal lesions caused by fighting among mice is the same type as that which colonizes the skin of the NOA mouse.

6) Various types of S. aureus are found in the fur of rats, but none of them produce superantigens.

These basic findings obtained at our facility indicate that the types of $S$. aureus that can be resident or transiently present in the fur (or skin) of mice are limited and these types of $S$. aureus also colonize the wet skin lesions of NOA mice.

It is known that DS-Nh mice spontaneously develop skin lesions (moist dermatitis) similar to the wet skin lesions in NOA mice, and $S$. aureus is believed to be one of the causes $[3,4]$. In addition, the $S$. aureus isolated from the skin lesions and skin surface of DS$\mathrm{Nh}$ mice is an SEC-producing strain similar to the isolates obtained from the skin of NOA mice, and the presence of antibody against SEC has also been demonstrated [17]. These findings for the DS-Nh mouse also indicate that the type of $S$. aureus that can colonize the skin (or fur) of the mouse is limited. The development of skin lesions in the DS-Nh mouse is restricted to the upper half of the body $[3,4]$, so the DS-Nh mouse can be clearly distinguished from the NOA mouse.

Due to the characteristic susceptibility of the skin of the NOA mouse to colonization with $S$. aureus, the usefulness of the NOA mouse as an animal model for evaluating the effects of topical antiseptics, using this bacterium as an index, was considered. As mentioned above, the colonization density of $S$. aureus in the skin of the NOA mouse increases with advancing age. Therefore, aged animals would appear to be the most useful as an animal model. However, wet skin lesions develop spontaneously in the NOA mouse from approximately 10 weeks of age, and from about 20 weeks of age, the skin lesions become more severe [8]. For these reasons, the use of animals older than 20 weeks may be inappropriate. The results of the present study show that individual differences in the colonization density of $S$. aureus decreased with advancing age. Accordingly, we used NOA mice at about 15 weeks of age as an animal model for evaluating the effects of topical antiseptics according to the present protocol. The time for evaluation of the antiseptic effects was set as $3 \mathrm{~h}$ after application, because our preliminary studies (unpublished data) showed that once bacteria were collected with TGSE agar from mouse skin, the recovery of bacterial counts would be insufficient until $3 \mathrm{~h}$ after collection.

In assessing the usefulness of the NOA mouse as an animal model for evaluating the effects of topical antiseptics, significant decreases were observed in $S$. aureus counts in tests using CHG-containing ointment and lotion $(P<0.05)$, in comparison with their corresponding bases. These results suggest that the effects of topical 
antiseptics can be evaluated using the characteristic susceptibility of the skin of the NOA mouse to colonization with $S$. aureus. However, in the case of ointments, the bacteria collection rate after application is affected by the covering effect of the test article, as was observed in the present study. Further investigations regarding this point should be conducted to establish a suitable evaluation system.

To date, the effects of topical antiseptics have been evaluated in vivo using a method in which an evaluation system employing an animal model prepared by experimental treatment is used. In this evaluation system, a skin infection disease model is obtained by burning, abrading, or cutting the dorsal skin of a rodent and inoculating the resulting wound with an indicator bacterium $[7,14,16]$.

The evaluation system we propose here exploits a characteristic of the skin of the NOA mouse and does not require experimental treatment. It is therefore a unique evaluation system. The use of naturally resident bacteria as an indicator should permit the effects of topical antiseptics to be evaluated under conditions closer to those in actual clinical practice. The similarity of the indicator bacterium S. aureus to the type that colonizes the eczematous lesions in humans with AD is thought to be particularly significant. Needless to say, the conventional skin infection model is useful for evaluating the effects of topical antiseptics targeting a specific pathogen or dermatopathy.

When the NOA mouse is maintained under ordinary breeding conditions after the application of a CHGcontaining ointment or lotion, $S$. aureus colonizes the skin again at high density (data not shown). Therefore, experimental animals may be used repeatedly. Furthermore, if the wet skin lesions that develop with age are employed, it should be possible to evaluate the effects of antiseptics and drug forms against the bacterial contamination of external wounds and bedsores without the need to inflict artificial burn-wounds or abrasions. The possibility of exploiting the particular characteristics of the skin of the NOA mouse to evaluate the effects and drug forms of topical antiseptics is therefore also advantageous from the viewpoint of animal welfare.

In our studies to date, we have shown that the NOA mouse is a new mutant with a range of potential uses [8-11]. In addition, we have presented here a novel characteristic of the NOA mouse (namely, that $S$. aureus readily colonizes the skin) and have demonstrated the possible usefulness of the NOA mouse as a model animal for evaluating the effects of topical antiseptics by exploiting this characteristic. However, we do not feel that we fully understand the characteristics of the NOA mouse. The NOA mouse will be marketed by CLEA Japan, Inc. (Tokyo, Japan) and has the potential for a wide range of applications. We expect that the NOA mouse will be studied in various areas and will contribute to the advancement of a number of fields in the future.

The abstract of this report was presented at the 51st Annual Meeting of the Japanese Association for Laboratory Animal Science (2004, Nagasaki, Japan).

\section{References}

1. Dahl, M.V. 1983. Staphylococcus aureus and atopic dermatitis. Arch. Dermatol. 119: 840-846.

2. Hanifin, J.M. 1984. Atopic dermatitis. J. Allergy Clin. Immunol. 73: 211-222.

3. Haraguchi, M., Hino, M., Tanaka, H., and Maru, M. 1997. Naturally occurring dermatitis associated with Staphylococcus aureus in DS-Nh mice. Exp. Anim. 46: 225229.

4. Hikita, I., Yoshioka, T., Mizoguchi, T., Tsukahara, K., Tsuru, K., Nagai, H., Horikawa, T., Tsuruta, Y., Suzuki, R., Ichihashi, M., and Horikawa, T. 2002. Characterization of dermatitis arising spontaneously in DS-Nh mice maintained under conventional conditions: another possible model for atopic dermatitis. J. Dermatol. Sci. 30: 142-153.

5. Ito, T., Ito, M., Utumi, K., Kagiyama, N., Saito, M., Takakura, A., Tanaka, T., and Nakagawa, M. 1988. Manual of Microbiologic Monitoring of Laboratory Animals (ICLAS Monitoring Center (Asia) eds.), Japanese Society of Laboratory Animals, Tokyo (in Japanese).

6. Kanzaki, H., Ueda, M., Morishita, Y., Akiyama, H., Arata, J., and Kanzaki, S. 1997. Producibility of exfoliative toxin and staphylococcal coagulase types of Staphylococcus aureus strains isolated from skin infections and atopic dermatitis. Dermatology 195: 6-9.

7. Kawabata, S., Masada, H., Wakebe, H., Ohmori, K., and Tamaoka, H. 1989. Bacteriological evaluation of OPC-7251, a new pyridone carboxylic acid antimicrobial agent: 2 . therapeutic effect of OPC-7251 cream on experimental infection model in mice. Chemotherapy 37: 1179-1183 (in Japanese).

8. Kondo, T., Shiomoto, Y., Kondo, T., and Kubo, S. 1997. The NOA mouse: a new hair-deficient mutant: a possible animal model of allergic dermatitis. Mouse Genome 95: 698-700.

9. Kondo, T., Shiomoto, Y., Kubo, S., and Kyogoku, M. 1999. NOA mouse: A new strain of mouse with a possibility of animal model for allergic dermatitis. Exp. Anim. 48: 315. 
10. Kondo, T., Kondo, T., Shiomoto, Y., and Momii, A. 2005. Effects of housing conditions on the development of wet skin lesions in the NOA mouse. Exp. Anim. 54: 149-154.

11. Kondo, T., Ohno, H., Kondo, T., Shiomoto, Y., and Momii, A. 2005. Development of dry skin in the NOA mouse under individual housing conditions: A potentially useful animal model for evaluating moisturizing effects. Exp. Anim. 54: 429-436.

12. Leyden, J.J., Marples, R.R., and Kligman, A.M. 1974. Staphylococcus aureus in the lesions of atopic dermatitis. Br. J. Dermatol. 90: 525-530.

13. McFadden, J.P., Noble, W.C., and Camp, R.D.R. 1993. Superantigenic exotoxin-secreting potential of staphylococci isolated from atopic eczematous skin. Br. J. Dermatol. 128: 631-632.

14. Nakae, N. and Inaba, H. 2000. Effectiveness of electrolyzed oxidized water irrigation in a burn-wound infection model. J. Trauma 49: 511-514.

15. Tada, J., Toi, Y., Akiyama, H., Arata, J., and Kato, H. 1996. Presence of specific IgE antibodies to staphylococcal enterotoxins in patients with atopic dermatitis. Eur. $J$. Dermatol. 6: 552-554.

16. Yamada, H., Hirao, M., and Kawabata, S. 2000. Evaluation of the in vivo drug efficacy of nadifloxacin cream: therapeutic effect in mouse skin infections disease models. Iyakuhin Kenkyu 31: 519-524 (in Japanese).

17. Yoshioka, T., Hikita, I., Matsutani, T., Yoshida, R., Asakawa, M., Toyosaki-Maeda, T., Hirasawa, T., Suzuki, R., Arimura, A., and Horikawa, T. 2003. DS-Nh as an experimental model of atopic dermatitis induced by Staphylococcus aureus producing staphylococcal enterotoxin C. Immunology 108: 562-569. 